\title{
escrito poŕm.
}

\section{'Jeniffer Johana Martínez Medina}

Estudiante doctoral, Escuela Graduada de Trabajo Social

Beatriz Lassalle, Universidad de Puerto Rico.

$\triangle$ jeniffer.martinez2@upr.edu

(D) ORCID ID https://orcid.org/0000-0003-0660-7711

\section{Elithet Silva Martínez}

- Catedrática Asociada, Escuela Graduada de Trabajo Social Beatriz Lassalle, Universidad de Puerto Rico.

$\triangle$ elithet_silva@upr.edu

(D) ORCID ID https://orcid.org/0000-0001-9688-1049

\section{Jennifer Oliveras Del Río}

- Auxiliar de Investigación de la Escuela Graduada de Trabajo

Social Beatriz assalle, Universidad de Puerto Rico.

$\rrbracket$ jennifer.oliveras@upr.edu

(D) ORCID ID https://orcid.org/0000-0001-6093-9034

\section{${ }^{4}$ Suzette Álvarez Soto}

Escuela Graduada de Trabajo Social Beatriz Lassalle,

Universidad de Puerto Rico.

\suzette.alvarez@upr.edu

(D) ORCID ID https://orcid.org/0000-0001-7853-3796

Martínez Medina, J. J., Silva Martínez, E., Oliveras Del Río, J., \& Álvarez Soto, S. (2020). Educación con perspectiva de género desde el diálogo entre las experiencias y saberes infantiles. Voces desde el

Trabajo Social, 8(1), 88-107. https://doi.org/10.31919/voces.v8i1.219

Recibido / received:

1 de septiembre de 2020
Revisado / reviewed:

20 de diciembre de 2020
Aceptado / accepted: 29 de diciembre de 2020

Derechos de autoría / Copyright: (c) 2020 Martínez Medina, J. J. et al. Este es un artículo de acceso abierto y distribuido bajo los términos de la licencia y políticas de Creative Commons Attribution 4.0 International License. 


\section{EDUCACIÓN CON PERSPECTIVA DE GÉNERO DESDE EL DIÁLOGO ENTRE LAS EXPERIENCIAS Y SABERES INFANTILES}

Jeniffer Johana Martínez Medina', Elithet Silva Martínez², (D) Jennifer Oliveras Del Río ${ }^{3}$, Suzette Álvarez Soto ${ }^{4}$

f openaCCESS PeER-REVIewed

\section{Resumen}

Este artículo enfatiza en la educación con perspectiva de género, mediante una investigación cualitativa en torno a los derechos y la dignidad de las niñas, llevada a cabo con estudiantes de la escuela elemental, promoviendo el diálogo con nuestros participantes, identificando conocimientos sobre dicha temática. La recopilación de información se hizo en tres fases, utilizando un lenguaje y estrategias pedagógicas acordes a su edad: 1) taller lúdico; 2) sesión artística de elaboración de dibujos sobre el tema investigado; 3) grupos focales para profundizar sobre género y equidad. Se evidenciaron discursos y prácticas basados en prejuicios y creencias aprendidas, como parte del sistema binario hombre/mujer que le atribuye superioridad al varón, y que inciden en su autoconcepto e interacciones. Simultáneamente, se destacó la capacidad creativa que despliegan para sustraerse y cuestionar tales visiones limitantes, y expresar libremente su ser/pensar/actuar. Entre los aspectos compartidos por quienes participaron, se destaca su pretensión de promover solidaridad y respeto por los derechos y la dignidad en igualdad de condiciones; el reconocimiento de la diferencia como seres humanos y su valor intrínseco inviolable; la injerencia de la niñez en aportar en todos los escenarios de socialización en los que se desenvuelven: hogar, 
comunidad, escuela. Lo evidenciado, sienta un precedente como ejercicios educativos, que contribuye en fomentar un sistema de valores alterno hacia la erradicación de la violencia y buenas prácticas de convivencia.

\section{Palabras claves}

educación, pedagogía lúdica, género, equidad

\section{Sumario}

Introducción. Metodología. Resultados. Discusión. Reflexiones finales. Recomendaciones. Referencias.

\section{[EN] EDUCATION WITH A GENDER PERSPECTIVE BASED ON THE DIALOGUE BETWEEN CHILDREN'S EXPERIENCES AND KNOWLEDGE}

\section{Abstract}

This article emphasizes education with a gender perspective, within qualitative research on the rights and dignity of girls, carried out with boys / girls from elementary school, promoting dialogue with the participants, and collectively identifying knowledge about this topic. The information gathered was performed in three phases, using language and pedagogical strategies according to their age: 1) workshop using play; 2) artistic session to elaborate drawings on the investigated topic; 3) focus groups to deepen on gender and equity. Discourses and practices based on prejudices and learned beliefs were evidenced, as part of the male / female binary system that attributes superiority to men, and that affect their self-concept and interactions. At the same time, the creative capacity they display to withdraw and question such limiting visions, and freely express their being / thinking / acting, was highlighted. Among the aspects shared by the participants, it stands out their claim to be promoters of solidarity and respect for the rights and dignity of all on equal terms; the recognition of difference as human beings and their unbreakable intrinsic value; the participation of children in contributing to all the socialization scenarios in which they engage 
in: home, community, school. This evidence sets a precedent in the matter of educational exercises, which contributes to promoting an alternative value system towards the eradication of violence and good coexistence practices.

\section{Keywords}

Education, recreational pedagogy, gender, equity

\section{Introducción}

Actualmente persisten diversas formas de inequidad entre hombres y mujeres a nivel mundial, permitiendo que las violencias contra las mujeres $y$ niñas estén presentes en los espacios públicos y privados de los que forman parte (ONU Mujeres, 2013). Esas inequidades se sostienen bajo un sistema de organización patriarcal que otorga a los hombres el poder en múltiples estructuras sociales. El patriarcado, así como los supuestos de género, son construcciones sociales que permean la organización de todos los ámbitos de la vida, imponiendo mandatos injustos hacia los géneros. El género es un conjunto de características que la sociedad asigna a las personas de forma diferenciada como propias de hombres o de mujeres, las cuales se concretizan mediante las estructuras sociales tales como: la familia, la formación educativa, la religión o el uso del lenguaje (Hendel, 2017). Bajo la categoría género se asignan comportamientos "apropiados" a las personas, según el sexo asignado al nacer. Estos roles de género construidos socialmente, han naturalizado la idea de que los hombres son superiores a las mujeres, y que dicha superioridad sirve de base para sostener una estructura de dominación, desigualdad e injusticia. Siguiendo a Martínez (2016a), para eliminar estos procesos de socialización arraigados a la cultura de género es necesario deconstruir $y$ establecer formas alternativas de conocimientos y prácticas, de manera que la equidad y la justicia se antepongan a las inequidades, el sufrimiento y las violencias. En ese sentido, la educación es una herramienta primordial como remedio contra el patriarcado y los supuestos injustos de género.

La Organización de las Naciones Unidas incorporó la perspectiva de género como una de las estrategias mundiales fundamentales para fomentar la igualdad de los géneros, mediante la Plataforma de Acción de Beijing durante la Cuarta Conferencia Mundial de las Naciones Unidas sobre la Mujer en el año 1995. La perspectiva de género permite visibilizar la realidad que viven las 
mujeres, así como los procesos culturales de socialización que internalizan y refuerzan mecanismos de subordinación de éstas (Facio \& Fries, 2005). Solís (2016), establece que la educación con perspectiva de género implica la formación de las personas desde la premisa básica de equidad entre los géneros, de forma que éstas puedan tener acceso igualitario a los servicios de educación libres de discriminación. $\mathrm{Si}$ bien es en el hogar y desde las familias donde la niñez obtiene sus primeros saberes, es a través de la educación formal donde se fortalecen y se ponen en práctica en primera instancia, dicho aprendizaje (Grimaldo \& Cervera, 2016). En ese sentido afirmamos que "la escuela como agente de socialización tiene el encargo de transmitir saberes, formar valores y establecer normas de comportamiento, proyectándose hacia modelos no sexistas y más democráticos" (Solís, 2016, p. 98). Estudios han señalado que, desde los seis años las niñas suelen considerarse menos brillantes 0 capaces que los niños (Bian et. al, 2017). Por esta razón, se hace necesario fomentar espacios que permitan concienciar sobre las capacidades y potencialidades de las niñas, de manera que éstas puedan desarrollar sus destrezas y asumir roles de liderazgo en los diversos ámbitos de su vida. Así la educación con perspectiva de género constituye una herramienta indispensable en la formación de las niñas para tal efecto, reconociendo a las escuelas primarias como instancias esenciales para erradicar las inequidades por razón de género.

La historia sobre la integración de la perspectiva de género a la política educativa de Puerto Rico tiene una larga trayectoria. Ésta no se da en el vacío, pues los avances en su favor han sido impulsados y promovidos particularmente por las luchas de movimientos de mujeres defensoras de los derechos humanos y la equidad. En el año 1972 fue aprobado el Título IX de las Enmiendas de Educación, legislación bajo la cual se prohíbe el discrimen por razón de sexo, en los programas y actividades de instituciones educativas de todos los niveles que reciban fondos federales. Posterior a la aprobación de dicha política, en Puerto Rico se realizaron dos investigaciones por Molini y Picó en la década de los 70 , que develaron el sexismo contenido en los libros educativos utilizados en las aulas (Martínez, 2016b). En ese sentido, se dieron a la tarea de desarrollar contenido educativo dirigido al personal docente y estudiantes de manera que se promoviera una política de equidad por sexo, acorde con el Título IX (Martínez, 2016b). Para el 30 de junio de 1999 fue aprobada la 
"Ley Orgánica del Departamento de Educación Pública de Puerto Rico" (Ley número 149), la cual favorecía la autonomía escolar, sin embargo, no incluyó ninguna legislación favorable a la equidad de género. En el 2006 fue aprobada la Ley número 108, la cual realiza una enmienda a la Ley Orgánica del Departamento de Educación del 1999. Con esta enmienda se proveía el espacio para que el Departamento de Educación junto con la Oficina de la Procuradora de las Mujeres, bajo el liderato de la Lcda. María Dolores Fernós, diseñaran un currículo educativo con el propósito de promover la equidad de género y prevenir la violencia contra las mujeres y niñas. Para la implementación de dicha ley, el Departamento de Educación con quien era secretario Rafael Aragunde, establece la Carta Circular Número 3 del 2008-2009. Bajo la misma se proponía la garantía de incorporar de forma transversal la perspectiva de género en el currículo escolar en el sistema público del país. A estos fines la carta describía la perspectiva de género así:

...herramienta pedagógica, al examinar las relaciones de género, buscará despojar los estereotipos que reflejan esas relaciones y transformar los contenidos de socialización a través de proyectos educativos específicos que fomentaran la igualdad entre los géneros, la justicia, la libertad, la paz, la convivencia solidaria, la participación democrática y el respeto a la diversidad humana. (Carta Circular núm. 3-2008-2009, p. 2)

Los movimientos de oposición a la perspectiva de género en el ámbito escolar comenzaron a ejercer presión. La resistencia se fundamentaba en la premisa de que educar desde la perspectiva de género a la niñez y la juventud, implicaba automáticamente orientarles hacia la homosexualidad y la promiscuidad sexual (Rodríguez, 2011). Sin embargo, al revisar lo descrito en la Carta Circular, la finalidad era la eliminación de la violencia y el discrimen por género en sus diversas manifestaciones, además de la expansión de la noción de derechos ciudadanos a los derechos de las mujeres, niños, niñas, personas negras, inmigrantes, con diversidad funcional $y$ otros grupos que históricamente han sido excluidos (Carta Circular núm. 3-2008-2009, p. 2). Entre los esfuerzos definidos en el plan de implementación se encontraba el desarrollo de estrategias $y$ metodologías educativas para incorporar la equidad de género en la educación, capacitación del personal sobre la perspectiva de género de forma transversal, su integración en el contenido curricular, identificación de contenido sexista en textos y materiales docentes para eliminar 
sus manifestaciones, al igual que la inserción del lenguaje inclusivo, entre otros (Carta Circular núm. 3-20082009). Poco tiempo después, el Departamento de Educación de Puerto Rico, bajo el liderato del Secretario Carlos Chardón, dejó sin efecto la Carta Circular.

En el año 2014, el secretario del Departamento de Educación, Rafael Román, emitió la Carta Circular número 19 del 2014-2015, que retomaba la política pública sobre la integración de la perspectiva de género en las escuelas públicas de Puerto Rico. La propuesta era muy similar a lo acotado en la anterior Carta Circular. Para su implementación se diseñó un currículo con el programa: "Salud Escolar: Manual Equidad de Género, en ruta hacia la construcción de un nuevo paradigma educativo" (Departamento de Educación de Puerto Rico, diciembre 2015). Este programa constituyó una propuesta de trabajo sistemático que vinculaba la comunidad escolar, bajo el valor rector de la equidad, definiendo estándares y expectativas para la prevención y control de la violencia, así como contribuyendo al desarrollo integral de las personas que participan como educandos, y que se superen las limitantes que por razón de género han sido impuestas por la sociedad (Departamento de Educación de Puerto Rico, diciembre 2015).
A pesar de la propuesta alternativa y de avanzada, en el año 2016, ésta fue derogada. La Secretaria de Educación, Julia Keleher, formuló la Carta Circular núm. 32 del 20162017, con relación a la "política sobre la equidad de género" en las escuelas del país, pero con un contenido muy general, que no dejaba claro en qué consistía. En el documento se establece que el Departamento "reitera su compromiso con promover la equidad de género, pero evitando establecer conceptos que generan confusión y donde no se respeten la diversidad de ideas y convicciones de nuestra sociedad" (Carta Circular Núm. 32-2016-2017, p.1).

Académicas en el campo de la educación han señalado que la eliminación de la perspectiva de género respondió a intereses político-partidistas, bajo el mando del entonces Gobernador Ricardo Rosselló, en el que se reflejaba desconocimiento en el tema (Yudkin en Tejada, 2017). Nuevamente, se frustró la implementación de la propuesta de integrar la perspectiva de género al currículo de enseñanza en las escuelas públicas del país. Esto a pesar de que la necesidad era apremiante y de que ciertamente, las leyes son fundamentales para hacer posibles los cambios sociales $y$ ofrecer un marco para desarrollarlos, mostrando el camino a seguir, aunque por si mismas no son garantía de 
que se lleve a cabo la transformación esperada (Rebollo, 2013). Si bien la aprobación de esta enmienda a ley resultó ser un gran avance en política educativa, la historia ha demostrado que tal logro resultó insuficiente, ante la falta de voluntad de la administración gubernamental para su implementación.

Precisamente por lo antes expuesto, esta investigación es fundamental en el proceso de aunar esfuerzos, movilizar recursos, desarrollar programas, implementar políticas y en el monitoreo de intervenciones encaminadas a la eliminación de violencias contra las mujeres y niñas (ONU Mujeres, 2013). Siguiendo a Grimaldo y Cervera (2016), investigar sobre la equidad de género en la educación primaria es prioritario para conocer cómo las niñas y los niños interpretan los roles de género de cada uno, obteniendo así un análisis de la realidad. Para facilitar dicho proceso y desarrollar junto con la niñez, conocimientos relacionados con la equidad de género, fueron diseñadas estrategias pedagógicas adecuadas a la etapa de desarrollo de quienes participaron en este estudio.

\section{Metodología}

Esta investigación cualitativa se propuso auscultar desde el diálogo de saberes en la infancia, para rescatar en los niños y las niñas participantes, su calidad de agentes interactivos en la educación, en aras de que se oriente con perspectiva de género. Las estrategias utilizadas se desarrollaron en varias fases: sensibilización, educación y posterior recopilación de información. La fase de sensibilización incorporó: 1) Un taller lúdico que incluía dinámica de armonización y creación, donde cada participante se representaba a sí mismo(a) y de auto valoración a través de su reflejo en un espejo; 2) la narración histriónica del cuento infantil "Las niñas serán lo que quieran ser" (Díaz, 2018), para comprender el alcance del género sobre las experiencias de vida de cada participante, y motivarles a reflexionar sobre la equidad de género.

Una fase intermedia fue llevada a cabo por el profesor de arte de la Escuela Elemental de la Universidad de Puerto Rico. Se realizó una sesión de arte y pintura, donde los y las participantes diseñaron bocetos que luego plasmaron en canvas, para ilustrar en sus obras una representación auténtica y creativa con relación al género, la solidaridad y equidad. En diálogo e interacción constante con el educador, todos/as lograron desarrollar sus pinturas, las cuales fueron exhibidas públicamente en la Plaza de Mercado de Río Piedras, sector urbano del municipio de San Juan, en conmemoración al 
Día Internacional de las Niñas, con el apoyo de la galería de arte que allí mismo se ubica, adscrita al Museo de Arte Contemporáneo de Puerto Rico (MACPR).

Finalmente, a los y las participantes se les organizó al azar en cuatro grupos, para el desarrollo de grupos focales, con fundamento en preguntas preestablecidas, que permitieron profundizar en temas asociados al género y la equidad.

En el presente estudio se cumplió con todos los principios éticos de protección de sujetos/as humanos/ as en la investigación, contando con la aprobación del protocolo No. 1920-014 por el Comité Institucional de Protección a Sujetos Humanos en la Investigación (CIPSHI), de la Universidad de Puerto Rico, Recinto de Rio Piedras, salvaguardando los derechos y el bienestar de los/as participantes en esta investigación.

Fueron 21 participantes entre niños y niñas, quienes desde el inicio se vincularon activamente en el proceso investigativo, reflexionando y expresándose creativamente a partir de sus propios pensamientos y vivencias, la percepción del género, los valores y creencias asociados al mismo, así como lo relacionado al ejercicio de sus derechos, y la necesidad de brindarse apoyo reciproco para tal efecto. Toda la información recopilada por escrito como resultado del taller lúdico, y los registros de audio de los grupos focales, fue transcrita, sistematizada y codificada con la utilización del programa ATLAS.ti, para identificar las categorías de análisis.

\section{Resultados}

La educación liberadora para indagar pensamiento-acción infantil sobre equidad de género

Una educación liberadora con perspectiva de género no es meramente el ejercicio de unas personas sobre otras, pues no se puede pensar por los demás, ni para los demás, pero tampoco sin ellos(as). Por esto es necesario que se reconozca la capacidad de todo ser humano para pensar por sí mismo/a y con los/as demás, y también para actuar (Freire, 2005) desde la infancia, y que la educación noconstituya unadoctrinamiento para el mantenimiento de mentalidades sumisas que reproducen modelos y patrones de opresión y violencia.

Al utilizar una pedagogía alternativa lúdica como mecanismo de acercamiento, con el desarrollo de talleres, se halló que: la niñez demandan en todo momento ser escuchada y comprendida, desde las particularidades de sus entornos, y no asumida como meros sujetos pasivos supeditados a la decisión y autoridad de la adultez, porque no 
es posible la transformación social hacia la equidad de género, si no se les involucra en esta dimensión que les impacta, desde el pensamiento crítico, auténtico, liberador y creativo. Diseñar y realizar actividades de juego como mecanismo de acercamiento y ejercicio para compartir con la infancia, es la mejor forma de materializar consideración y respeto hacia la niñez. De ahí la necesidad de plantearse respuestas pedagógicas que les brinden herramientas alternativas, priorizando el desarrollo equitativo de sus capacidades sin cohibición, sin infundirles miedo, y estimulando la libre expresión creativa de su sentir y ser. Esto demandó en la presente investigación, configurar las condiciones que promuevan un presente digno y respetuoso de la infancia, de su autonomía para pensar y crecer, que simultáneamente es una clara práctica de justicia social. Se motivó desde el salón de clases ejercicios que les integran, reconociendo lo diferentes que son, logrando incentivando a que sus procesos educativos, contribuyan efectivamente a instituir unos referentes públicos éticos que restablezcan la dignidad de todo ser humano, por el valor intrínseco a su existencia. Todo ello permitió trascender del mero cambio de retórica y de lenguaje, para resignificar simbólica y prácticamente la inclusión de la diversidad como principio pedagógico (Solís, 2016).

Quedó develado que la aproximación mediante juegos, más allá de reproducir información, motiva la generación de saberes y permite a la niñez manifestarse desde su sentir, sobre el mundo que les rodea. Este debiera ser un foco de atención fundamental en sus procesos educativos. Sin duda, hay en la educación recreativa, una práctica concreta de educación liberadora, instituida en el principio del respeto hacia la dignidad humana, el fortalecimiento de la expresión autónoma del ser, y valorar como oportunidad natural de la existencia la diversidad de otredades.

\section{Equidad de género}

Se narró dramatúrgicamente el cuento "Las niñas serán lo que quieran ser" (Díaz, 2018), cuya historia es alusiva a los obstáculos que enfrentan las niñas por la inequidad del género y cómo ello impacta en la reproducción de estereotipos que coartan su libertad para pensar y ser. Esta herramienta artística no sólo instó a la libre expresión infantil sobre el género y sus estereotipos, a veces reafirmándolos y en otras cuestionándolos, sino que además promovió la participación para plantear distintas propuestas de cómo realmente desde la adultez, pueden apoyarles en sus procesos 
de desarrollo, para ser más felices, sentirse con mayor seguridad y construir un mundo mejor, como se ejemplifica a continuación en sus verbalizaciones:

"A mí me gustaría que nadie estuviera maltratando a la gente ni nada"; "Que el mundo sea feliz y que todo el mundo se respete especialmente a las niñas"; "es que sí, somos diferentes porque nos vemos diferentes, pero somos iguales porque podemos ser lo que queramos sin importar si eres un nene o una nena, y por eso no te tienen que prohibir hacer algo que te gusta"; "Ser niña para mí es estar siempre feliz y nunca me gustaría rendirme, porque me gustaría cumplir mi sueño para poder ser una gran científica".

El cuento fue una excelente estrategia de ambientación previa, para posteriormente realizar con el docente de artística, la sesión de diseño de bosquejos y plasmar los dibujos en canvas, relacionados con equidad de género y solidaridad. Hay en cada obra de arte todo un conjunto de conocimientos, que vale la alegría retomar. Sin embargo, para efectos de este artículo, se exponen a continuación algunas verbalizaciones explicativas sobre estos:

"Mi dibujo es una niña que está volando en el cielo, y que ella es la única, diferente y aquí dice: todas las niñas son diferentes, pero no significa que tú eres fea, gorda, flaca o mala. Tú eres como eres y nadie te puede cambiar ni ofender. Tú vas a lograr tus metas y nadie te lo va a impedir. Así que levanta tu cabeza y sigue hacia adelante"; "Para mi ser niña o niño es tener paz, amor, amistad, respeto, un corazón derecho, amigos, paciencia, responsable y también ser tierna. Entonces aquí yo escribí las niñas tienen un montón de cosas adentro de ellas, igual que los niños".

Un niño socializa su obra así: "mi dibujo es que está un niño en la calle y luego se encuentra a una niña... eso fue el día pasado, que se conocieron y el niño dijo: ¿quieres ser mi amiga? Y ella dijo que sí. El día siguiente se pudieron ver y la niña dijo: yo soy única, bonita y yo puedo ser lo que quiera ser. Él pudo entender eso, entonces fueron amigos...y fueron amigos para siempre".

Con el firme propósito de ampliar el diálogo con quienes participaron, los niños y niñas estuvieron presentes en una sesión en la que al azar fueron organizados en cuatro grupos focales, cada uno acompañado de una investigadora, quienes orientarían la discusión siguiendo las preguntas abiertas preestablecidas. Se identificó que a temprana edad, ya son portavoces de un discurso propio de igualdad para ser lo que deseen, y manifiestamente narran cómo independientemente de cómo se identifican, les puede agradar 
mucho actividades o colores que habitualmente no son asignados a su género, y que a veces se contienen por la incomodidad de sentir que eso que les atrae, puede no ser bien visto ni aceptado por el resto de las personas. Claras evidencias de ello fueron verbalizadas en los siguientes términos:

Una niña recalca:

"A veces si juegan en algunos deportes las nenas, pues tienen que jugar con los nenes que porque ellos son mejores".

Argumento respecto del cual difiere un niño:

"pero eso no es cierto, las nenas pueden jugar lo que ellas quieran".

Continuando el debate otro niño expone:

"yo creo que hay cosas como dijo ella, que las niñas no pueden hacer, cosas que pueden hacer los hombres, pero también los hombres no pueden hacer cosas que las niñas pueden hacer. Como por ejemplo, maquillarse, los hombres... (hace una pausa y guarda silencio), reanuda el participante que hizo la pausa anterior: "pero eso no se puede hacer porque no se vería... bien".

Finalmente, otro participante transa el debate manifestando:

"pero eso era antes, antes no se podía hacer eso porque era una ley, pero ahora todo cambió y ahora si un niño de verdad quiere, si se pueden poner cosas de las niñas"; "es raro cuando una niña se pone pantalones. A pesar de todo a mí no me gustan las faldas, y siempre me gustan los pantalones, pero es una pelea con mi mamá para que me vea muy nena, pero yo tampoco me quiero ver tan nena porque no me gustan las faldas"; "los colores no tienen sexo, porque algunos niños dicen que los colores azules, naranja y todas esas cosas son solamente de ellos y los rositas son de niñas. Tenemos el derecho a escoger los colores que queramos".

Esa genuina libertad infantil, de la que aún hay mucho en sus esencias del ser, coexiste simultáneamente con las creencias y roles que devienen del entorno social, en cuanto a un deber ser que les encierra en la dualidad hombre/mujer, masculino/ femenino. Como se ejemplifica por ellos/as al manifestar:

"Lo bueno de ser niña es que puedo hacer lo que quiera y lo malo es que tienes que limpiar mucho"; "una ventaja pequeña que yo tengo por ser niño es que físicamente soy como que más fuerte que las niñas, pero una desventaja es que últimamente las niñas están recibiendo más ayuda que los niños"; "es que los niños siempre se burlan de las niñas porque son diferentes y porque son únicas".

Los prejuicios cercenan esa originalidad de ser y hacer, que al ir calando hondo y adoptando expresiones más amplias en su 
proceso de crecimiento en el trasegar de la vida, si no se les visibiliza y cuestiona como han logrado hacerlo en este ejercicio investigativo, se traducen en expresiones múltiples de violencias de género, incluidas las que se ejecutan contra las diferentes identidades que salen del binarismo. Se evidencia en la concepción de niños y niñas, el matiz diferenciador entre sí, de acuerdo con lo que se espera de cada cual. Así, los niños se conciben en un posicionamiento superior a las niñas por tener más fuerza y una amplia gama de deportes que demandan mayor rendimiento físico, a diferencia de las niñas que han de optar de manera más restringida por los deportes restantes de menor fuerza física, según lo manifestado.

La restricción de accesos de las niñas a ciertos juegos es una exclusión latente, y simultáneamente el preludio de negación de aspiraciones a las futuras adultas. De igual forma, el encasillamiento de los niños que les niega elegir un favorecimiento por actividades señaladas como propias para las niñas, les coarta la posibilidad de la afinidad por estas actividades y también inhibe aspiraciones hacia la adultez. Esto permite visualizar que, la equidad de género no es un asunto meramente de feminismos o de las mujeres, como suele ser etiquetado, es un tema y un aspecto transversal de vida que atañe a todos y todas, porque somos interdependientes. Al propiciarnos condiciones de bienestar con equidad y respeto recíproco indistintamente de las creencias y preferencias, lograremos mejorar conjuntamente, como expuso un participante: "todos tenemos los mismos derechos, todos podemos ser lo que queramos ser".

\section{Discusión}

Un aspecto neurálgico presente en todo el proceso investigativo, y de manera esencial en el acercamiento y comunicación dialógica sostenida con la infancia, es la educación con perspectiva de género. Justamente por eso, se informa a través de las distintas dinámicas y juegos, la conceptualización del género como algo ajeno a su ser natural, algo con lo que no se nace. En este sentido, el género es producto de una construcción social no asociada naturalmente aunque si discursivamente, a factores biofísicos que se relaciona con el sexo (Varoucha, 2014). El género es representado socio-culturalmente, mediante parámetros que impactan la vida de las personas, obedeciendo a un sistema de valores para establecer roles y expectativas de comportamiento humano (Varoucha, 2014).

Por eso, el género está vinculado a la percepción externa del conjunto social, lo cual incluye una fuerza 
vinculante en la medida que se ajuste a la ortodoxia de los referentes del sistema normativo social, o segrega a quienes los transgreden. De ahí, que esa dimensión transversal de la vida que no nace con nosotros/as, sí nos marca desde el nacimiento, incluso justificado en los aspectos relacionados con el sexo, para legitimar discursos manipulados $y$ presentarlos como incuestionables, configurándose en prejuicios que filtran la autodefinición del ser. Son esos estereotipos, creencias limitantes que están desplegando multiplicidad de formas de violencia y sufrimiento humano, tales como: discriminación, maltrato infantil y explotación física, múltiples manifestaciones de violencia y feminicidios. Todo esto amerita ser visibilizado, cuestionado y erradicado desde los procesos educativos, como ejercicios de consciencia y reflexión permanente, optando por pedagogía lúdica afín a la edad de niños y niñas.

¿Por qué en la actualidad, como se ha diseñado la educación formal es una equivocación tan socialmente extendida y aceptada, frente a la equidad del género y cualesquiera factores de clasificación de las personas? Pertenecemos a sociedades altamente burocratizadas, organizadas jerárquicamente, y divididas tajantemente entre quienes planifican y quienes ejecutan, quienes mandan y quienes obedecen, sin que se establezcan entre sí roles basados en relaciones solidarias y coordinadas de trabajo hacia un bienestar común. Suele insistirse en la delegación personal que conduce a la enajenación, despersonalización y al no haber una apropiación consciente de lo social, lo público y lo político de parte de las personas, las relaciones son determinadas, por agentes externos. Esto pareciera una abstracción, a veces indescifrable y amorfa, pero estuvo manifiestamente presente en este ejercicio investigativo, visible en sus consecuencias y condicionamientos. Se evidencia cómo las desigualdades de género parecieran persistir bajo el control de una especie de mano invisible, y cómo la ciudadanía va reproduciendo y ejecutando condiciones que ordenan referentes a seguir, aunque no emanen concretamente de la voluntad de una persona en particular (Zuleta, 2001).

La escuela tiene una marcada función política, se esperaría que se instituya como saber hacer reflejado en acción, y la materialización de ideas fecundas producidas gracias al conocimiento, para la construcción integral de pensamiento-acción y convivir mutuamente en el respeto por las libertades (Arendt, 2016), despojándose de ideas excluyentes. Así, el proceso educativo sería un 
ejercicio creativo y el aprendizaje una diversión, no una labor tortuosa.

Manifiestamente la función socializadora de la escuela consiste en educar personas, transmitiéndoles ciertos conocimientos y capacidades que necesariamente debe desarrollar en relación con la cultura. Existen también otras funciones a veces menos obvias, como las de reproducir discursos oficiales del Estado frente a la autoridad, lo público, el ejercicio de poder y la aplicación de las normas. No obstante, se debe reconocer que gracias a este espacio los y las estudiantes aprenden a tener cierto nivel gradual de independencia emocional en relación con la familia, inmiscuirse dentro de la esfera pública, experimentar con mayor profundidad las relaciones interpersonales y afrontar conflictos cotidianos en los que decidir desde su autonomía.

\section{Reflexiones finales}

Cuando se omite directamente la alusión al género en el proceso educativo desde la infancia, ya les está transmitiendo elementos de invisibilización, trivialización, desconocimiento de una realidad que es inherente y transversal a la existencia humana social, con un fuerte impacto en sus identidades subjetivas. Si desde la infancia se gestan ya las diferencias injustas y hostiles entre hombres y mujeres, obviando que incluso hay masculinidades y feminidades múltiples, además de una gama diversa de identidades no binarias, se les está bloqueando la posibilidad de comprender realidades sociales, tanto como la de conocerse y reconocerse. De ahí, la necesidad de reflexionar con la infancia sobre las concepciones de género, porque ello ciertamente ahora y a futuro es determinante en sus relaciones e interacciones sociales, destacando que existen aspectos en común que nos vinculan y comunican: la equidad, la convivencia, la comunicación dialógica para el entendimiento; y que las diferencias entre las personas no son razón para establecer indicadores de inferioridad o superioridad, para su categorización jerárquica. Algo como esto, vigente en nuestras sociedades, es una falacia agresiva, altamente perjudicial contra la dignidad humana, pues tenemos el mismo valor intrínseco por el sólo hecho de existir.

Ciertamente, la educación con perspectiva de género desde la infancia lleva implícito un componente de posicionamiento ideológico y político para hacer frente desde la educación a la opresión y violencia. Esta educación liberadora e inclusiva enlaza perspectivas críticas, comprensivas, configura alternativas frente a la cómoda incomodidad de lo que acontece en 
el sistema de género discriminatorio, implementado socio-culturalmente. Entonces, será viable, no sólo analizar sino exponer y denunciar las complejas y arraigadas expresiones de opresiones ancladas desde nuestro pasado histórico, reforzadas en el presente, y combatir los efectos nocivos de seguirla reproduciendo a futuro.

En este proceso investigativo logramos develar que mediante pedagogía lúdica es altamente posible coeducar y cocrear con la niñez, a partir de un acercamiento desde la comunicación dialógica, fundada en confianza, solidaridad y participación, propiciandolas mismas posibilidades y oportunidades de ser escuchados/as, de reafirmar como importantes sus aportaciones, sueños, expectativas, aspiraciones, desmantelando procesos de asimilación sexistas.

Aunque se escucha una amplia propaganda de respeto e igualdad como referentes de valoración entre las personas, en la práctica cotidiana estonoescoherenteconlos prejuicios, estereotipos, creencias transmitidas a la infancia, naturalizando las desigualdades en actividades, roles, y responsabilidades, que no tendrían por qué ser adjudicadas a un grupo en particular, menos fundamentado en el género. Es importante a través de la educación, vincular a todos y todas como corresponsables del bienestar propio, tanto como el de las demás personas, generando mejores condiciones de convivencia a nivel familiar, escolar, laboral, social, estatal, indistintamente de nuestra identidad socio-cultural, creencias, que son producto de construcciones. No se ha de menospreciar ni obviar que la niñez con su gran capacidad de reflexión y aportación, también requieren mayores apoyos y acompañamiento en estos procesos de cocreación social, para proveerles protección y seguridad, pero incentivando su autoconfianza y autoestima.

\section{Recomendaciones}

Nos urge como sociedad el reaccionar de manera proactiva ante la desproporción en feminicidios y en el número de mujeres víctimas de violencia en nuestro país. En nuestras manos se encuentra atajar las violencias, no exclusivamente de manera remediativa, sino de manera preventiva. Estos esfuerzos deben incluir la eliminación de las inequidades por género en todas las esferas de la vida, incluyendo la esfera educativa. Para lograr coherencia con el compromiso de desarrollar personas plenas en la sociedad, se deben retomar las recomendaciones de múltiples organismos internacionales, personas expertas en el tema y organizaciones que trabajan por 
los derechos humanos, de considerar una reforma educativa hacia la equidad como fundamento para promover relaciones humanas saludables. Existe vasta literatura que sustenta como vital la incorporación de una perspectiva de género hacia la equidad desde la formación primaria. Tal y como lo expone Solís (2016), esta incorporación no sólo traerá beneficios al ámbito escolar, sino también al desarrollo de competencias de vida. Es importante repasar que cuando se habla de competencias de vida, se busca que, desde las edades tempranas, se preste atención de manera intencionada y específica a crear espacios pedagógicos para transformar patrones valorativos hacia el respeto de las diferencias de género, de modo que podamos formar personas con consciencia ética y respetuosa de los derechos humanos de las demás (González \& Villaseñor, 2010).

No basta con sugerir en retórica la inclusión de modelos curriculares hacia la equidad de género. Urge traer a la mesa a todas las personas que componen el sistema educativo con el fin de trabajar de manera participativa y reflexiva sobre los temas en cuestión. Esto no puede lograrse si no se proporcionan espacios de diálogo participativo, sensibilización y concienciación sobre los alcances de la inequidad de género en nuestra sociedad. En este sentido, es vital afianzar en esta ecuación el concepto de comunidad escolar, enfatizando en la importancia que tienen los y las docentes, el personal no docente, madres, padres y personas cuidadoras, en conjunto con las y los estudiantes. De manera simultánea, recomendamos espacios intencionados sólo para educadoras y educadores, en los que se pueda trabajar con contenido y procesos en temas de implementación de un currículo con perspectiva de género.

Si bien es importante reconocer que en el entorno escolar se hace necesario tener líneas de trabajo claras e inclusivas de todas las personas que lo componen, no podemos ignorar la relación que tiene la escuela con la familia y la comunidad de donde proviene el estudiantado. Un modelo para considerar es el que presenta Díaz (2009) hacia prevención de la violencia de género. En el mismo, sugiere el concepto de "una nueva escuela", en el que se considere la escuela, como fundamental para servir de puente con las familias, al reconocer que la formación escolar puede muy bien alinear la educación en valores y en equidad de género. Por esa misma línea Rubio (2009) plantea que, si se ve a la escuela como centro de aprendizaje de habilidades y destrezas para la vida, 
también asumiría un rol primordial en educar para una vida social justa y equitativa.

Definitivamente es necesario hablar de co-responsabilidad en lo referente a la formación para la vida de nuestros niños, niñas y jóvenes. Por un lado, se requiere presencia de los padres, madres y personas cuidadoras, y por otro se requiere un compromiso ético político de todo un sistema formal educativo, para poder darnos a la tarea de construir otras maneras de relacionarnos y de vivir. Esto sin dejar de mirar toda la estructura social en la que se enmarcan las violencias, opresiones e inequidades que nos atraviesan como integrantes de una sociedad cimentada por un sistema patriarcal, capitalista y colonial.

\section{Referencias}

Arendt, Hannah. (2016). La promesa de la política. Paidos.

Bian, L., Leslie, S., \& Cimpian, A. (2017). Gender stereotypes about intellectual abilitymerge early and influence children's interests. Science. 355, 389-391.

Departamento de Educación. (2008). Carta Circular Núm. 3:20082009: Declaración de política pública sobre la incorporación de la perspectiva de género en la educación pública puertorriqueña. San Juan, Puerto Rico.

Departamento de Educación. (2015). Carta Circular Núm. 19:20142015: Política Pública sobre la equidad de género y su integración al currículo del Departamento de Educación de Puerto Rico como instrumento para promover la dignidad del ser humano y la igualdad de todos y todas ante la ley. San Juan, Puerto Rico.

Departamento de Educación de Puerto Rico. (Diciembre 2015). Programa de salud escolar, Manual equidad de género. En ruta hacia la construcción de un nuevo paradigma educativo. San Juan, Puerto Rico. 
Departamento de Educación.

(2017). Carta Circular Núm. 32:2016-2017: Política Pública sobre la Equidad de Género y su integración al currículo del Departamento de Educación de Puerto Rico como instrumento para promover la dignidad del ser humano y la igualdad de todos y todas ante la ley. San Juan, Puerto Rico.

Díaz, R. (2018). Las niñas serán lo que quieran ser, ( $1^{\mathrm{a}}$ ed.), España.

Díaz, M. (2009). Convivencia escolar y prevención de la violencia de género desde una perspectiva integral. Revista del Consejo Escolar del Estado: Participación Educativa, 11, 59-72.

Facio, A., \& Fries, L. (2005). Feminismo, género y patriarcado. Revista sobre enseñanza del derecho de Buenos Aires, 6, 261-281. http://www.derecho.uba.ar/ publicaciones/rev academia/revistas/06/ feminismo-genero-y-patriarcado.pdf

Freire, Paulo. (2005). Pedagogía del oprimido. Siglo XXI.

González, M., \& Villaseñor, M. (2010). La perspectiva de género en el sistema educativo de Jalisco: Bases para la acción. Revista de Educación y Desarrollo, 14(1), 23-29. http:// www.cucs.udg.mx/revistas/edu desarrollo/ anteriores/14/014 Villasenor.pdf
Grimaldo, A., y Cervera, C. (2016). Equidad de género en la educación primaria. Construcciones y deconstrucciones. Jóvenes en la Ciencia, 2(1), 764-768.

Hendel, L. (2017). Perspectiva de género. Fondo de las Naciones Unidas para la Infancia (UNICEF). Argentina. https://www.unicef.org/ argentina/sites/unicef.org.argentina/files/201804/COM-1 PerspectivaGenero WEB.pdf

Ley Núm. 108 para añadir un inciso (bb) al Artículo 6.03 de la Ley Núm. 149 de 15 de junio de 1999, según enmendada, conocida como la Ley Orgánica del Departamento de Educación, a los fines de imponer la obligación al Departamento de Educación de diseñar e implantar un currículo dirigido a promover la equidad por género y la prevención de violencia doméstica en coordinación con la Oficina de la Procuradora de las Mujeres. (26 de mayo de 2006).

Martínez, L. (2016b). Políticas educativas sobre género en Puerto Rico: una historia de retos. Raudem, Revista de Estudios de las Mujeres. http:// dx.doi.org/10.25115/raudem.v4i0.1738 
ONU Mujeres. (2013). Elementos esenciales de planificación para la eliminación contra la violencia de mujeres y niñas. http://www. endvawnow.org/uploads/modules/ pdf/1372349315.pdf

Rebollo, M. (2013). La innovación educativa con perspectiva de género. Retos y desafíos para el profesorado. Revista de Currículum y Formación de Profesorado, 17(1), 3-8. https://www. redalyc. org/articulo.oa?id=567/56726350001 http://kalathos.metro.inter.edu/kalathos mag/ publications/archivo1 vol5 no1.pdf

Rubio, A. (2009). El abandono y la falta de éxito de los chicos en la escuela, un problema de género. Revista del Consejo Escolar del Estado: Participación Educativa, 11, 23-39.

Solís, A. (2016). La perspectiva de género en la educación. En Trujillo, J. A. y García, J. L. (coords.). Desarrollo profesional docente: reforma educativa, contenidos curriculares y procesos de evaluación (pp. 97-107). Escuela Normal Superior. http://www.rediech.org/inicio/ images/k2/Desarrollo2-articulo2-5.pdf
Tejada, A. (2017, 24 de febrero). Educación con perspectiva de género: "un asunto de derechos humanos". Diálogo UPR. https://dialogoupr.com/educacioncon-perspectiva-de-genero-un-asuntodederechoshumanos/?fbclid=IwAR3i7 VOKBuWt mhFV3TauFROiatvIC7Ln 5mUEfifYS-QxLBC4LKdfjkFLb8K

Varoucha, E. (2014). La identidad de género, una construcción social. Mito revista cultural, pp. 1-5. http://revistamito.com/la-identidad-degénero-una-construcción-social//

Zuleta, E. (2001). Elogio de la dificultad y otros ensayos ( $5^{a}$ Ed.) Fundación Estanislao Zuleta, Cali, Colombia. 